\title{
sciendo
}

DOI: $10.2478 / f v-2020-0017$

\section{THE EFFECT OF CO-ADMINISTRATION OF INACTIVATED VACCINES AGAINST BOVINE RESPIRATORY DISEASE AND NEONATAL CALF DIARRHOEA}

\author{
Makoschey, B. ${ }^{1}$, Vertenten, G. ${ }^{1}$, Reddick, D. ${ }^{2}$ \\ ${ }^{1}$ Intervet International BV/MSD-Animal Health, Wim de Körverstraat, 5831 AN Boxmeer \\ The Netherlands \\ ${ }^{2}$ Moredun Scientific, Pentlands Science Park, Bush Loan, Penicuik, Midlothian, EH26 OPZ \\ United Kingdom
}

geert.vertenten@merck.com

\section{ABSTRACT}

A pilot study was performed to evaluate the safety and serological responses after co-administration of two multivalent inactivated vaccines to pregnant cattle. One vaccine was directed against bovine respiratory disease (BRD) and contained antigens of bovine respiratory syncytial virus (BRSV), parainfluenza 3 virus (PI3) and Mannheimia haemolytica (Mh). The second vaccine targeted neonatal calf diarrhoea (NCD) and was composed of inactivated antigens of bovine rotavirus (BRV), bovine coronavirus (BCV) and $E$. coli. The use of these combinations have been used more and more by veterinary practitioners as there exist some clear evidence that both vaccines improves the passive protection via the colostrum for the relevant pathogens. However, up until now, no safety or efficacy data has been available concerning such co-administrations. The safety of both vaccines and the serological responses to the $B R D$ vaccine has been evaluated when used at the same time, but without mixing and compared to the responses to the administration of each vaccine independently. There was no evidence of any negative effect on calving or calf health in any of the vaccinated animals. The antibody levels against BRSV and $\mathrm{Mh}$ in the sera of the calves from cows vaccinated with both vaccines were not significantly different from the levels in the sera of calves vaccinated with the BRD vaccine alone. The results from this pilot study demonstrated that the co-administration of the two multivalent inactivated vaccines had no detrimental effect on the safety or serological responses to the $B R D$ vaccine compared to the independent use of the vaccines.

Key words: bovine respiratory disease complex; cow vaccination; neonatal calf diarrhoea; passive immunity

\section{INTRODUCTION}

Neonatal calf diarrhoea (NCD) and bovine respiratory disease (BRD) are the most important health problems in calves during the first month of life $[2,13]$. Both diseases are multifactorial and co-infections with two or more pathogens are common. The list of pathogens associ- 
ated with NCD includes bovine rotavirus (BRV), bovine coronavirus (BCV), E. coli and Cryptosporidium parvum $[1,5,17]$. In the case of BRD, the prevalence vary between studies, but bovine respiratory syncytial virus (BRSV) and Mannheimia haemolytica (Mh) seem to be most often involved [8].

In addition to the cost of mortality and treatment of the sick calves, the economic consequences of calf-hood diseases may include reduced growth performance, as well as increased age and difficulty at first calving and reduced milk yields $[14,15,16]$. Given the increased public pressure to reduce intensive antimicrobial use in farm animals, vaccination is an efficient measure to reduce the use of antibiotics in the treatment and prevention of BRD and NCD.

The importance of colostrum has not only been demonstrated for the protection against NCD, but also against BRD [19]. Cow vaccination is widely applied for the prevention of NCD and more recently, the beneficial effects against BRD has been reported $[9,11,12]$.

In order to obtain the highest antibody levels in the colostrum, the cows must be vaccinated shortly before calving. The possibility for the co-administration of a BRD and a NCD vaccine would simplify the herd vaccination schedules and reduce the handling of the animals.

In general, no information is available on the safety and efficacy of the co-administration of separately licensed vaccines. Therefore, regulators advise that a decision needs to be made on a case by case basis. In the pilot study reported here, the safety and serologic response to BRSV and Mh after co-administration of two multivalent inactivated vaccines to pregnant cattle was investigated. One vaccine was directed against BRD and contained antigens of BRSV, parainfluenza 3 virus (PI3) and Mh. The second vaccine targeted NCD and was composed of inactivated antigens of BRV, BCV and E. coli. The antibody levels against PI3 were not measured, as any negative effects from the co-administration on the serological response to the $\mathrm{BRD}$ vaccine is likely to be more pronounced for the other two antigens measured [10].

\section{MATERIALS AND METHODS}

The United Kingdom Home Office controls experiments and scientific procedures which may have the effect of causing the animals pain, suffering, distress or lasting harm and does so by the issue of licenses under the Animals (Scientific Procedures) Act 1986. Moredun Scientific Limited conducts its work under Home Office License Numbers PPL/60/3884 and PPL/60/3747. These licenses strictly specify the limits of severity of effects on the animals.

\section{Study design}

The purpose of this study was to evaluate the safety and BRD specific serological responses of two commercial inactivated multivalent vaccines for cattle when administered concurrently to pregnant cattle. In addition, an assessment of the safety of the co-administration to pregnant animals was carried out, with additional observations on the vaccinated animals' offspring.

\section{Animal Husbandry}

The animals (Holstein Friesian) were housed at a conventional dairy farm in accordance with the standard husbandry practices. The study animals were co-mingled and housed in the same accommodation as non-study animals. The cows were fed a complete mixed diet for pregnant dairy cows (mixture of grass silage, whole crop wheat, brewers' grain, concentrate pellets and molasses). Animals in lactation received a ration of concentrates at each milking. Free access to hay/silage and clean water was available at all times.

Prior to Day 0, thirty pregnant cows were allocated to the treatment groups (10 per treatment group), ensuring that each treatment group contained an even spread of animals with a range of BRSV antibody titres (based on prestudy screening). Calves were left with their mothers for the first 24 hours following birth and then fed with commercial milk replacer.

\section{Vaccinations}

All vaccinations were given approximately 3 to 6 weeks prior to the expected calving dates (D0). Animals in test group 1 (T01) were administered $2 \mathrm{ml}$ of a vaccine against neonatal calf diarrhoea (NCD). The vaccine contained inactivated antigens of bovine rotavirus (BRV), bovine coronavirus (BCV) and E. coli (Rotavec ${ }^{\circledR}$ Corona; MSDAnimal Health, Boxmeer, The Netherlands) and was applied by intramuscular injection into the proximal area of the neck. Animals in test group 2 (T02) were vaccinated subcutaneously with $5 \mathrm{ml}$ of an inactivated bovine respi- 
ratory disease (BRD) vaccine containing a BRSV-PI3-Mh combination (Bovilis ${ }^{\oplus}$ Bovipast RSP; MSD-Animal Health, Boxmeer, The Netherlands). The injections were given into the distal area of the neck. Animals from test group 3 (T03) were concomitantly administered $2 \mathrm{ml}$ of the NCD vaccine by intra-muscular injection into the proximal area of the neck, and $5 \mathrm{ml}$ of the BRD vaccine by subcutaneous injection into the distal area of the neck at a site distinct from the site where the other vaccine was applied. All injections were administered to the right side of the neck. Commercial vaccine batches released according to the standard requirements of the manufacturer were used.

\section{Evaluation of safety parameters}

Clinical observations and injection site assessments were carried out following the administration of the vaccines. The observations consisted of a measurement of the rectal temperature from Day 2 to Day 4 and an assessment of the general condition of the animals from Day 2 to Day 4 by experienced personnel once daily, and by a veterinarian prior to vaccination on Day 0 . The injection site assessments were carried out by experienced personnel once daily for all study animals from Days 0 to Day 7, and then twice weekly until the reactions had resolved (up to Day 28) or upon calving. The injection site assessments consisted of the following observations at the injection sites and surrounding area: presence of swelling, size of swelling measured by a calibrated ruler ( $\mathrm{mm}$ : length $\times$ breadth), and the type of swelling (e.g. oedema, firmness). The calves were observed within 24 hours of birth, to determine whether they were free from abnormalities and were in good general health.

\section{Measurement of BRSV and Mh antibody responses}

Non-heparinized blood samples were collected from each cow: prior to administration, within 24 hours after calving, and from each calf within 24 hours after birth. The antibody levels against BRSV and Mh were determined by quantitative ELISA. The BRSV assay was an in-house ELISA. The M. haemolytica A1 antibody ELISA was performed as described previously [10].

First, all plates were coated. The positive and negative detergents extracted coating antigens were diluted in ELISA coating buffer ( $\mathrm{pH}$ 9.6) and mixed well. The working dilution of the coating antigens is detailed in Table 1 and was pre-determined by a checkerboard titration.
Table 1. Working dilution of the coating antigens in antibody BRSV ELISA test

\begin{tabular}{llc}
\hline & Reagent & Dilution \\
\hline Coating Antigen & Positive: BRSV Rispoval & $1 / 800$ \\
& Negative: BRSV BEK Control & $1 / 800$ \\
Positive Control & M989 & $\begin{array}{c}\text { High 1/100 } \\
\text { Low 1/1100 }\end{array}$ \\
Negative Control & FBS & $1 / 50$ \\
Conjugate & Rabbit a Bovine HRP & $1 / 5000$ \\
Substrate & Enhanced K-Blue TMB & Neat \\
Stop Solution & 0.25 M Sulphuric Acid (H2SO4) & Neat \\
Coating Buffer & $\begin{array}{l}\text { Sodium Carbonate/Bicarbonate } \\
\text { Buffer pH 9.6 }\end{array}$ & N/A \\
Wash Fluid & 1xPBS/0.05\% Tween 20 (PBST) & N/A \\
Diluent & $\begin{array}{l}\text { 1xPBS/Tween 20/EDTA/0.55\% } \\
\text { ovalbumin (PBSTOval) }\end{array}$ & N/A \\
\hline
\end{tabular}

$100 \mu \mathrm{l}$ of the positive antigen was added to all of the wells in rows A, B, E and F of a medium binding ELISA plate (Greiner M129A) and $100 \mu$ of negative antigen to all of the wells in rows $\mathrm{C}, \mathrm{D}, \mathrm{G}$ and $\mathrm{H}$. The coated plate was sealed and incubated overnight at $+2{ }^{\circ} \mathrm{C}$ to $+8^{\circ} \mathrm{C}$. The ELISA diluent (PBST/Ovalbumin) was prepared by adding $2 \mathrm{ml}$ of $0.1 \mathrm{M}$ EDTA to $178 \mathrm{ml}$ of ELISA wash fluid (PBST). Approximately $20 \mathrm{ml}$ of this solution was added to $1 \mathrm{~g}$ of ovalbumin powder in a universal and stirred to dissolve the ovalbumin. A Buchner funnel was prepared using Whatman No. 3 filter paper-filter was pre-wetted with some of the remaining PBST/EDTA solution and the $20 \mathrm{ml}$ ovalbumin solution was filtered through the filter and the filtrate was added to the remaining $160 \mathrm{ml}$ of PBST/EDTA solution. The ELISA coating antigen was discarded from the plate. The plate was washed 4 times with ELISA wash fluid (PBST) and the plate was blotted dry. The test serum was diluted 1:50 in the appropriate sample dilution tube by adding $10 \mu \mathrm{l}$ of sample to the pre-aliquoted $490 \mu \mathrm{l}$ of ELISA diluent (PBST/Ovalbumin). Positive and negative control sera were diluted in ELISA diluent (PBST/Ovalbumin). 100 $\mu \mathrm{l}$ of positive and negative control sera dilutions and $100 \mu \mathrm{l}$ of diluted test sample were added to the appropriate 4 wells ( 2 positive and 2 negative) of the coated ELISA plate. The plate was sealed and incubated for 1 hour ( +10 minutes) at $37^{\circ} \mathrm{C}\left(+2^{\circ} \mathrm{C}\right)$. The plate was washed 4 times with ELISA 
Table 2. Mean rectal temperature and percentage of cows with a rectal temperature exceeding $39.0^{\circ} \mathrm{C}$ before and after vaccination

\begin{tabular}{|c|c|c|c|c|c|c|c|}
\hline \multirow{3}{*}{ Group } & \multicolumn{7}{|c|}{ Days before/after vaccination } \\
\hline & -2 & -1 & 0 & 1 & 2 & 3 & 4 \\
\hline & \multicolumn{7}{|c|}{ Mean Rectal Temperatures $\left[{ }^{\circ} \mathrm{C}\right] /$ Percentage cows $>39.0^{\circ} \mathrm{C}$} \\
\hline NCD & $38.5 / 0$ & $38.5 / 0$ & $38.6 / 0$ & $38.7 / 0$ & $38.6 / 20$ & $38.4 / 0$ & $38.4 / 0$ \\
\hline BRD & $38.4 / 0$ & $38.6 / 0$ & $38.7 / 10$ & $38.8 / 30$ & $38.5 / 10$ & 38.50 & $38.5 / 10$ \\
\hline Co-administration & $38.5 / 10$ & $38.5 / 10$ & $38.6 / 10$ & $39.0 / 50$ & $38.6 / 10$ & $38.4 / 10$ & 38.40 \\
\hline
\end{tabular}

Mean rectal temperatures $\left[{ }^{\circ} \mathrm{C}\right]$ and percentage of calves with a rectal temperature exceeding $39.0^{\circ} \mathrm{C}$ before and after vaccination with an inactivated NCD vaccine (T01), an inactivated BRD vaccine (T02) or co-administration of both vaccines (T03)

wash fluid (PBST) and blotted dry. $100 \mu$ l of diluted Rabbit anti-Bovine Horseradish Peroxidise (RaBovHRP) conjugate in ELISA diluent (PBST/Ovalbumin) was added to all of the wells. The plate was sealed and incubated for $50-$ $70 \mathrm{~min}$ at $35-39^{\circ} \mathrm{C}\left(+2^{\circ} \mathrm{C}\right)$. The plate was washed 4 times with ELISA wash fluid (PBST) and blotted dry, $100 \mu \mathrm{l}$ of enhanced TMB substrate was added to all of the wells and incubated at room temperature for 5 minutes to allow the colour to develop. The reaction was stopped by adding $50 \mu \mathrm{l}$ of Stop solution ( $0.25 \mathrm{M}$ sulphuric acid) to all of the wells. The plate was read at $450 \mathrm{~nm}$.

The results of the samples were produced as a Dynex Revelation 4.04 printout. This consisted of a Data Matrix/Table; containing the corrected optical density values (ODs) and a Ratio Results table; containing the Ratio Matrix calculated results (Ratio Matrix). The positive and negative results were taken from the Ratio Matrix (RM) values as follows: $\mathrm{RM}$ value $<0.100$ was negative, $\mathrm{RM}$ value $\geq 0.100$ and $<0.150$ was equivocal (repeat/investigation of another sample approx. 14 days apart), and RM value $\geq 0.150$ was positive.

The BRSV and M. haemolytica antibody levels for the group vaccinated with the BRD vaccine (T02) were compared with the group vaccinated with both vaccines (T03) and with the group vaccinated with the NCD vaccine (T01). The formula for Student's T-test for two-tailed distribution and two-samples with unequal variance within Microsoft Excel were applied. In the results, the values are given as the mean \pm the standard deviation.

\section{RESULTS}

\section{Clinical reactions and body temperatures after vaccination}

No abnormal observations relating to the application of the vaccines were observed over the four day period postvaccination (Study Day 0 to Day 4). The body temperatures of some animals were slightly increased after vaccination but remained within the physiological range. Five of the 10 vaccinated animals in T03 had a temperature exceeding $39.0^{\circ} \mathrm{C} 1$ day after vaccination, but the temperature normalized $\left(<39.0^{\circ} \mathrm{C}\right)$ within 24 hours. See Table 2 for the mean group rectal temperatures and the percentage of cows with a rectal temperature exceeding $39.0^{\circ} \mathrm{C}$ before and after vaccination.

\section{Injection site reactions}

One animal in the group vaccinated with the NCD vaccine alone and one animal in the group vaccinated with both vaccines had measurable injection site reactions to the NCD vaccine (see Table 3 for the results). The reaction persisted until 28 days post vaccination in the case of vaccination with only one vaccine and disappeared within 24 hours post vaccination in the animal that was given both vaccines.

After the subcutaneous vaccination with the BRD vaccine alone, all 10 animals had a measurable injection site reaction for up to 21 days after vaccination as compared to 8 animals having measurable site reactions for up to 17 days after vaccination in the group that received both vaccines. 


\section{Calving results}

A total of 27 cows calved successfully after an average duration of pregnancy of 285,282 and 283 days for the groups vaccinated with the NCD vaccine, the BRD vaccine, or both vaccines, respectively. The three remaining cows gave birth to stillborn calves; one animal vaccinated with the BRD vaccine gave birth to a stillborn calf approximately three weeks before the expected calving date. The findings at the post mortem indicated that the abortion was most likely due to an infection with the Bacillus licheniformis. A second animal in that group gave birth to a still born calf. Findings at that post mortem examination indicated that the calf was born to term and the death was most likely caused by asphyxiation. Twin calves were stillborn slightly before term from one cow of the group vaccinated with both vaccines. No abnormalities were observed at that post mortem examination.

\section{Health conditions of calves born alive}

All calves born alive were found healthy and remained healthy during the four days observation period with the exception of one calf born from a dam vaccinated with both vaccines (individual data not shown). That calf had an increased respiratory effort from birth onwards. The demeanour was normal. However, on thoracic auscultation a tachycardia was apparent. The calf was euthanized, and a necropsy was performed. Gross findings showed evidence of pneumonia in all lobes of the lung with an abscess in the left antero-dorsal region. It was concluded that the animal was suffering from a pulmonary infection.

A

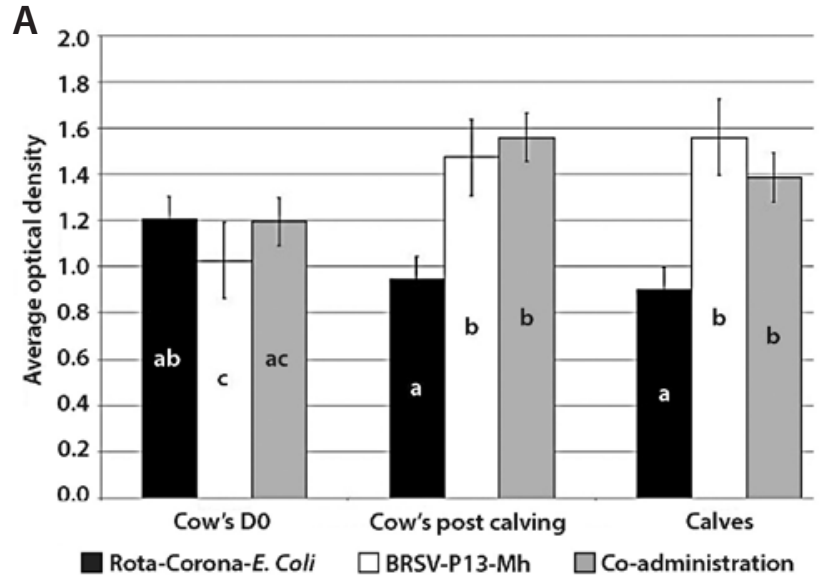

\section{Serological responses against BRSV and $\mathrm{Mh}$}

All cows were sero-positive for BRSV at the time of vaccination. The BRSV-specific IgG levels were significantly lower at the time of vaccination in cows vaccinated with BRD vaccine alone (T02 $1.027 \pm 0.182)$ compared to cows vaccinated with NCD vaccine alone (T01 1.210 \pm 0.188 ) $(p=0.04)$. There was no statistical difference between the mean BRSV-specific IgG levels of other treatment groups at the time of the vaccination (Figure $1 \mathrm{~A}$ for comparison of mean values) ( $\mathrm{p}=0.89$ and 0.12 for respectively T01 vs T03 and T02 vs T03).

The BRSV-specific IgG levels were significantly higher in post-calving cows vaccinated with BRD vaccine alone ( $\mathrm{p}=0.003$; T02 $1.474 \pm 0.396)$ or co-administration with the NCD vaccine ( $\mathrm{p}=0.05$; T03 $1.322 \pm 0.414)$ compared to cows vaccinated with NCD vaccine alone (T01 $0.950 \pm 0.285)$. Moreover, there was no statistical difference between the mean BRSV-specific IgG levels post calving of the cows vaccinated with the $\mathrm{BRD}$ vaccine alone compared to the co-administration ( $\mathrm{p}=0.44$ ) (see Figure 1A for comparison of average values).

All calves were positive for the detection of antibodies against BRSV with the exception of one animal in the group vaccinated with the NCD vaccine alone. However, the BRSV-specific IgG levels were significantly higher in calves born from cows vaccinated with the BRD vaccine alone ( $\mathrm{p}=0.02$; T02 1.561 \pm 0.383 ) or co-administered ( $\mathrm{p}=0.01$; T03 1.388 \pm 0.375 ) compared to calves born from cows that received only the NCD vaccine (T01 0.904 \pm 0.416 ). Moreover, there was no significant difference in the BRSV-spe-

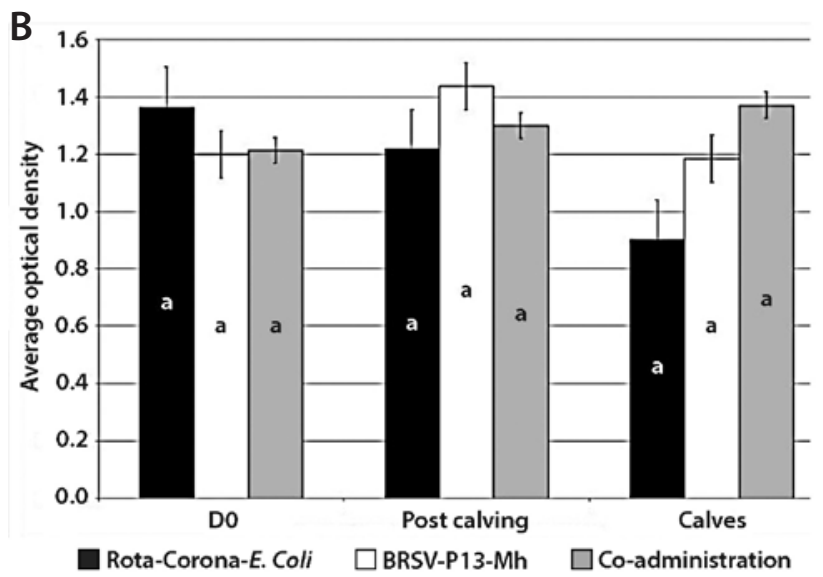

Fig. 1. Antibody response against BRSV and $\mathrm{Mh}$

Antibody response against BRSV (A) and Mh (B) expressed as OD values \pm standard error in the sera of the vaccinated animals at the time of vaccination, post calving and in the sera taken from their calves. The cows were vaccinated with an inactivated NCD vaccine (T01) (black bars), an inactivated BRD vaccine (T02) (white bars) or co-administration of both vaccines (T03) (grey bars) 
cific IgG levels of calves born from cows that received the $B R D$ vaccine alone or co-administered $(\mathrm{p}=0.32)$.

The results of the Mh antibodies were similar to those for the BRSV antibodies; they indicated a trend towards higher titres for the calves from cows vaccinated with both vaccines (T03 1.214 $\pm 0.372,1.299 \pm 0.346,1.371 \pm 0.481$ at respectively day of vaccination, post calving and in calves); however the results were not significantly different from the other groups (see Figure 1B for comparison of average values). All cows were sero-positive for Mh at the time of vaccination and after calving. All calves were positive for the detection of antibodies against $\mathrm{Mh}$ with the exception of the calf born to a cow that was vaccinated only with the NCD vaccine that was also negative for BRSV specific antibodies. There was no significant difference in Mh-specific IgG levels after the calving of cows that received the $\mathrm{BRD}$ vaccine alone (T02 $1.438 \pm 0.268$ ) or co-administered $(p=0.33$; T03 1.299 \pm 0.346$)$. Also for the other groups, there was no significant difference in the Mh-specific IgG levels in the cows after calving $(\mathrm{p}=0.18$ and 0.65 for respectively T01 $(1.220 \pm 0.410)$ vs T02 $(1.438 \pm 0.268)$ and T01 $(1.220 \pm 0.410)$ vs T03 $(1.299 \pm 0.346))$. There was no significant difference in the Mh-specific IgG levels of calves born from cows that received the $\mathrm{BRD}$ vaccine alone (T02 1.185 \pm 0.551 ) or co-administered (T03 1.371 \pm 0.481 ) $(p=0.46)$. Also for the other groups, there was no significant difference in the Mh-specific IgG levels in the calves $(\mathrm{p}=0.32$ and 0.07 for respectively T01 $(0.905 \pm 0.606)$ vs T02 (1.185 \pm 0.551$)$ and T01 $(0.905 \pm 0.606)$ vs T03 (1.371 \pm $0.481))$.

\section{DISCUSSION}

The pilot study presented here was performed to investigate the safety and efficacy of concomitant application of two multivalent inactivated vaccines in pregnant cattle. The results indicated that the two multivalent inactivated vaccines were tolerated well after co-administration of both vaccines. A transient rise in temperatures is a common observation after application of inactivated bacterial multi-strain vaccines $[3,6]$. Inactivated viral vaccines often do not induce hyperthermia [7].

In general, swellings may represent a local reaction against vaccine antigens and/or the adjuvant components contained in the vaccines. Reactions, as seen in this study, have been reported for different kinds of adjuvants [7, 20] and are generally considered as acceptable for cattle vaccines. Most importantly in the context of this study, the number and the duration of local reactions did not increase after concurrent use of both vaccines.

As the pilot study was performed in pregnant animals, the outcome of the pregnancy was the most important safety parameter. For all three cows that gave birth to dead calves, the cause was unlikely to be related to the vaccination. Similarly, it was concluded that the calf with health problems was suffering from either congenital pneumonia or pulmonary infection, both of which are not considered to be related to the vaccinations. Considering all of the safety results together, there were no detrimental effects after the single or concurrent use of the two inactivated multivalent vaccines in pregnant cattle.

The second objective of the study was to evaluate the efficacy of the vaccines when used singly or concurrently. The purpose of cow vaccination is to increase antibody levels in the cow which are then transferred to the calves via the colostrum $[4,18]$. Therefore, in the context of this study, the measurement of antibody levels in the calves' sera was an appropriate parameter to judge the efficacy of these vaccines as colostrum management was appropriate.

The results of BRSV and Mh antibodies in the calf sera clearly indicated that the vaccinations induced a specific immune response in the cows and, most importantly, the antibody response in the calf sera were not significantly different between the groups vaccinated with the vaccine against bovine respiratory disease alone or with both vaccines.

\section{CONCLUSIONS}

The results of this pilot study are promising, but further studies are required to fully demonstrate the safety and the efficacy after co-administration of these two vaccines and the value of passive immunity against respiratory pathogens.

\section{ACKNOWLEDGEMENT}

This study was funded by MSD Animal Health, Boxmeer, The Netherlands 


\section{CONFLICT OF INTEREST}

The authors Birgit Makoschey and Geert Vertenten declare conflict of interest. They are employees of MSD Animal Health, the company that markets the vaccines that have been used in the study reported herein.

\section{REFERENCES}

1. Bartels, C. J., Holzhauer, M., Jorritsma, R., Swart, W. A., Lam, T. J., 2010: Prevalence, prediction and risk factors of enteropathogens in normal and non-normal faeces of young Dutch dairy calves. Prev. Vet. Med., 93, 2-3, 162-169. DOI: 10.1016/j.prevetmed.2009.09.020.

2. Brscic, M., Leruste, H., Heutinck, L. F., Bokkers, E. A., Wolthuis-Fillerup, M., Stockhofe, N., et al., 2012: Prevalence of respiratory disorders in veal calves and potential risk factors. J. Dairy Sci., 95, 5, 2753-2764. DOI: 10.3168/jds. 2011-4699.

3. Cervino, M., Figueras, L., Martin, S., Elvira, L., Callus, M., Dowlut, S., et al., 2011: Specific humoral response and effect on rectal temperature of two clostridial vaccines in lambs. Vet. Rec., 168, 17, 458. DOI: 10.1136/vr.c6865.

4. Crouch, C. F., Oliver, S., Francis, M. J., 2001: Serological, colostral and milk responses of cows vaccinated with a single dose of a combined vaccine against rotavirus, coronavirus and Escherichia coli F5 (K99). Vet. Rec., 149, 4, 105-108. DOI: 10.1136/vr.149.4.105.

5. Garcia, A., Ruiz-Santa-Quiteria, J. A., Orden, J. A., Cid, D., Sanz, R., Gomez-Bautista, M., et al., 2000: Rotavirus and concurrent infections with other enteropathogens in neonatal diarrheic dairy calves in Spain. Comp. Immunol. Microbiol. Infect. Dis., 23, 3, 175-183. DOI: 10.1016/s01479571(99)00071-5.

6. Garcia de la Fuente, J. N., Gutierrez-Martin, C. B., Ortega, N., Rodriguez-Ferri, E. F., del Rio, M. L., Gonzalez, O. R., et al., 2004: Efficacy of different commercial and new inactivated vaccines against ovine enzootic abortion. Vet. Microbiol., 100, 1-2, 65-76. DOI: 10.1016/j.vetmic.2004.01.015.

7. Gethmann, J., Huttner, K., Heyne, H., Probst, C., Ziller, M., Beer, M., et al., 2009: Comparative safety study of three inactivated BTV-8 vaccines in sheep and cattle under field conditions. Vaccine, 27, 31, 4118-4126. DOI: 10.1016/j.vaccine.2009.04.072.

8. Grissett, G. P., White, B. J., Larson, R. L., 2015: Structured literature review of responses of cattle to viral and bacterial pathogens causing bovine respiratory disease complex. J. Vet. Intern. Med., 29, 3, 770-780. DOI: 10.1111/jvim.12597.

9. Kremer, P., Brunner, R., Nüske, S., Gruber, E. C., Scholz, A. M., 2010: Herd immunization as an approach to reduce the incidence of respiratory disease in calves: A case study. In Proceedings of the XXVI World Buiatrics Congress, Santiago de Chile, Chile, November, 14-18.

10. Makoschey, B., Chanter, N., Reddick, D. A., 2006: Comprehensive protection against all important primary pathogens within the bovine respiratory disease complex by combination of two vaccines. Praktische Tierarzt., 87, 10, 819-826.

11. Makoschey, B., Ramage, C., Reddick, D., Fraser, S., Donachie, W., 2012: Colostrum from cattle immunized with a vaccine based on iron regulated proteins of Mannheimia haemolytica confers partial protection. Vaccine, 30, 5, 969973. DOI: 10.1016/j.vaccine.2011.11.044.

12. Meyer, G., Cuquemelle, A., Delverdier, M., Ronsin, L., Gallard, Y., Teillaud, A., 2015: Protection des veaux contre le virus respiratoire syncytial bovin par la vaccination des vaches en fin de gestation (Protection of young calves against bovine respiratory syncytial virus by vaccination of the cows at the end of pregancy). In Journées Nationales GTV, 2015, Nantes, France, Mai 20-22, 771-775.

13. Pardon, B., Alliet, J., Boone, R., Roelandt, S., Valgaeren, B., Deprez, P., 2015: Prediction of respiratory disease and diarrhea in veal calves based on immunoglobulin levels and the serostatus for respiratory pathogens measured at arrival. Prev. Vet. Med., 120, 2, 169-176. DOI: 10.1016/j.prevetmed. 2015.04.009.

14. Schaffer, A. P., Larson, R. L., Cernicchiaro, N., Hanzlicek, G. A., Bartle, S. J., Thomson, D. U., 2016: The association between calfhood bovine respiratory disease complex and subsequent departure from the herd, milk production, and reproduction in dairy cattle. J. Am. Vet. Med. Assoc., 248, 10, 1157-1164. DOI: 10.2460/javma.248.10.1157.

15. Stanton, A. L., Kelton, D. F., LeBlanc, S. J., Wormuth, J., Leslie, K. E., 2012: The effect of respiratory disease and a preventative antibiotic treatment on growth, survival, age at first calving, and milk production of dairy heifers. J. Dairy Sci., 95, 9, 4950-4960. DOI: 10.3168/jds.2011-5067.

16. Trilk, J., Munch, K., 2008: Connections between health of calves, growth and later yields of dairy cattle. Zuchtungskunde, 80, 6, 461-472.

17. Uhde, F. L., Kaufmann, T., Sager, H., Albini, S., Zanoni, R., Schelling, E., et al., 2008: Prevalence of four enteropathogens 
in the faeces of young diarrhoeic dairy calves in Switzerland. Vet. Rec., 163, 12, 362-366. DOI: 10.1136/vr.163.12.362.

18. Weaver, D. M., Tyler, J. W., VanMetre, D. C., Hostetler, D. E., Barrington, G. M., 2000: Passive transfer of colostral immunoglobulins in calves. J. Vet. Intern. Med., 14, 6, 569-577. DOI: 10.1892/0891-6640(2000)014<0569:ptocii > 2.3.co;2.

19. Windeyer, M. C., Leslie, K. E., Godden, S. M., Hodgins, D. C., Lissemore, K. D., LeBlanc, S. J., 2014: Factors asso- ciated with morbidity, mortality, and growth of dairy heifer calves up to 3 months of age. Prev. Vet. Med., 113, 2, 231-240. DOI: 10.1016/j.prevetmed.2013.10.019.

20. Yeruham, I., Yadin, H., Haymovich, M., Perl, S., 2001: Adverse reactions to FMD vaccine. Vet. Dermatol., 12, 4, 197-201. DOI: 10.1046/j.0959-4493.2001.00221.x.

Received March 19, 2020

Accepted May 11, 2020 